\title{
Étude de la génération d'harmoniques par des faisceaux tronqués
}

\author{
S. Kazamias, F. Weihe, D. Douillet, C. Valentin, T. Planchon, S. Sebban, \\ G. Grillon, F. Augé, D. Hulin et Ph. Balcou \\ Laboratoire d'Optique Appliquée, Chemin de la Hunière, ENSTA, Ecole Polytechnique,
UMR 7639 du CNRS, 91761 Palaiseau cedex, France
}

\begin{abstract}
Résumé: Nous présentons une étude complète de l'influence de la diaphragmation du faisceau infrarouge sur la génération d'harmoniques d'ordres élevés. Nous avons mesuré l'efficacité de conversion obtenue dans différents gaz et pour différentes énergies laser en fonction de l'ouverture du diaphragme. Les courbes ainsi déduites présentent un caractère assez général : le maximum d'efficacité de conversion se détache nettement des valeurs obtenues pour des ouvertures de diaphragme trop importantes: un facteur 25 d'amélioration est obtenu entre le cas sans diaphragmation et le cas optimal. Une simulation basée sur un code uni-dimensionnel de calcul d'accord de phase dépendant du temps permet de reproduire fidèlement les résultats expérimentaux. Nous pouvons ainsi conclure que l'ouverture optimale du diaphragme est celle qui réalise le meilleur compromis entre la géométrie focale dont découle l'accord de phase (qui aurait tendance à favoriser des diaphragmes fermés) et l'amplitude et la phase du dipôle harmonique (qui sont favorisées par des diaphragmes ouverts). [1]
\end{abstract}

\section{INTRODUCTION}

Les harmoniques sont une source de rayonnement XUV qui peut être utilisée pour des expériences d'application si le flux de photons obtenu est suffisant. Ce flux peut être optimisé dans différentes configurations d'interaction laser-gaz : dans les capillaires, le laser est totalement guidé [2], ce qui permet de maintenir une intensité constante sur toute la longueur du milieu et donc un bon accord de phase. La configuration en jet ou en cellule de gaz permet plus de liberté dans le contrôle du faisceau et la géométrie du foyer joue ici un rôle fondamental. Pour optimiser les harmoniques, un diaphragme est souvent placé sur le faisceau avant focalisation dans le milieu gazeux [3] [4], ceci quand l'énergie contenue dans le laser est suffisante pour garantir des intensités focalisées supérieures à l'intensité de saturation (de l'ordre de quelques $10^{15} \mathrm{~W} / \mathrm{cm}^{2}$ ). Ce résultat est bien connu empiriquement mais n'avait jamais été complètement expliqué. Nous avons observé que les efficacités de conversion entre l'infrarouge et l' XUV peuvent être multipliées par un facteur allant jusqu'à 25 lorsque le diaphragme d'entrée est fermé à environ la moitié du waist initial du laser (facteur 10 sur le nombre de photons).

La diaphragmation induit de nombreuses modifications sur le laser : l'énergie transmise est diminuée, la zone focale est largement étendue longitudinalement et transversalement, ce qui induit une forte variation de l'éclairement, donc des taux d'ionisation mais aussi du dipôle et de la phase atomiques. Tous ces effets couplés influencent notablement l'accord de phase et par suite le nombre de photons harmoniques.

Nous avons donc modélisé nos observations expérimentales par un code de génération et de propagation d'harmoniques unidimensionnel qui prend en compte tous les paramètres énoncés ci-dessus. Notre spectrographe détecte l'émission harmonique sur l'axe et possède un faible angle solide de collection, ce qui explique que les résultats sont en très bon accord avec les mesures et nous permettent de mieux comprendre la physique complexe de la génération d'harmoniques. 


\section{DISPOSITIF EXPERIMENTAL ET EFFICACITES DE CONVERSION}

Nous générons les harmoniques en focalisant un laser Ti:Sa délivrant des impulsions de $6 \mathrm{~mJ}$ en $30 \mathrm{fs}$. La longueur d'onde centrale est de $800 \mathrm{~nm}$ et le waist initial du laser de $22 \mathrm{~mm}$. Le diaphragme est placé avant la lentille $(f=1 \mathrm{~m})$. Le milieu gazeux est constitué par une cellule de longueur variable percée par le laser lui-même en son foyer (trous de 100 à 200 microns). Le rayonnement harmonique est analysé par un spectromètre à transmission dont la calibration précise nous permet de donner des résultats en nombre absolu de photons par harmonique et par tir. Le maximum obtenu est de $10^{10}$ photons par harmonique et par tir dans l'argon vers $30-40 \mathrm{~nm}$, ce qui représente une efficacité de conversion de $3 \cdot 10^{-5}$.

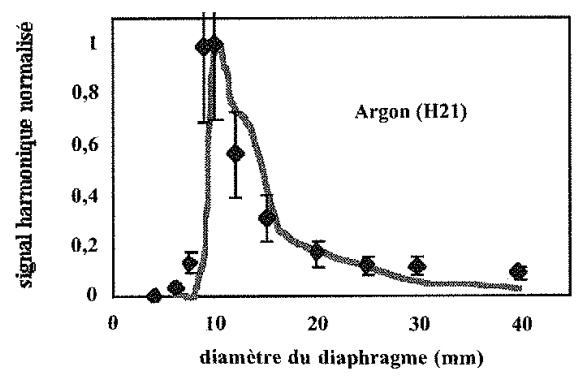

Figure 1. Signal expérimental de l'harmonique 21 dans l'argon en fonction de l'ouverture du diaphragme (carrés noirs). La ligne continue est le résultat du calcul théorique dans les mêmes conditions. (cellule de $2 \mathrm{~mm}$, pression 10 torr, foyer du laser en entrée de cellule, $6 \mathrm{~mJ}$ d'énergie totale)

La figure 1 montre le comportement caractéristique du flux harmonique en fonction de la taille du diaphragme. Le signal croît très rapidement jusqu'au maximum puis décroît plus doucement au fưr et à mesure que le diaphragme est ouvert. On retrouve le même comportement général pour le néon avec un diaphragme optimum de $11 \mathrm{~mm}$ au lieu de $10 \mathrm{~mm}$ pour l'argon en raison de la loi de coupure. Pour le xénon le maximum est moins brutal et s'observe pour des diaphragmes plus ouverts. On explique ce résultat par le fait que la dispersion atomique est plus importante, ce qui permet d'avoir un bon accord de phase pour des degrés d'ionisation plus élevés que pour les gaz légers.

\section{EFFETS GEOMETRIQUES DE LA DIAPHRAGMATION DU FAISCEAU}

Ces effets sont nombreux, le plus simple étant la variation de l'énergie transmise. Il ne faut cependant pas négliger la modification de la zone focale qui joue un rôle clé dans la génération d'harmoniques. En effet, la surface transverse du faisceau au foyer varie comme l'inverse du diamètre du diaphragme, tout comme la longueur de Rayleigh (dimension longitudinale). La première influence le volume dans lequel une certaine harmonique est générée et la seconde définit le gradient de la phase de focalisation (dite phase de Gouy) qui détermine en grande partie l'accord de phase. Enfin, l'intensité au foyer dépend très fortement de la taille du diaphragme (puissance quatrième pour les faibles ouvertures) et implique des taux d'ionisation très différents donc une modification de la propagation du rayonnement harmonique (longueur de cohérence très diminuée par l'ionisation), ainsi qu'un dipôle harmonique différent. C'est pourquoi nous avons réalisé une mesure soigneuse du front d'onde expérimental du laser infrarouge avant focalisation avec un 
Shack-Hartmann [5]. Un programme de propagation par diffraction de Fresnel nous permet d'avoir une connaissance précise de l'éclairement réel à une certaine distance du foyer. Le rapport entre la valeur expérimentale et la valeur obtenue avec un faisceau gaussien pur (dit rapport de Strehl) s'éloigne notablement de 1 pour des diaphragmes assez ouverts (voir figure ci-dessous):

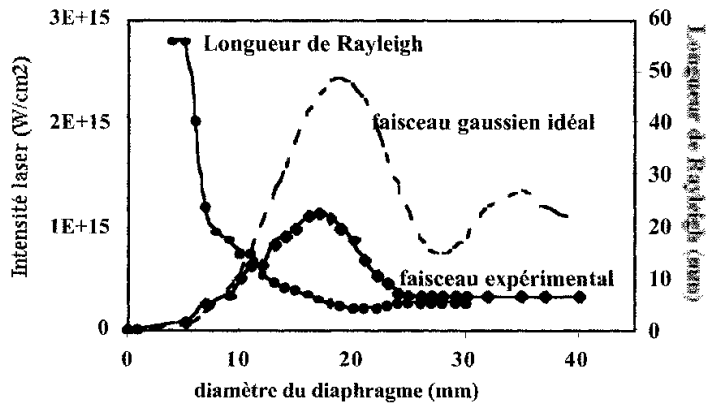

Figure 2. Variation de la longueur de Rayleigh du faisceau infrarouge diaphragmé (points), intensité expérimentale à $8 \mathrm{~mm}$ du foyer (carrées noirs) comparée à l'intensité qu'on obtiendrait avec un faisceau gaussien idéal de front d'onde plan (ligne pointillée).

Cette étude ne prend pas en compte les effets de défocalisation induits par l'ionisation progressive du gaz pour de forts éclairements laser. On remarque cependant que la génération d'harmoniques la plus efficace a lieu pour des intensités nettement inférieures à l'intensité de suppression de barrière pour les gaz considérés $\left(1-310^{14} \mathrm{~W} / \mathrm{cm}^{2}\right)$.

\section{CODE DE GENERATION D'HARMONIQUES ET PROPAGATION}

Notre code est basé sur le calcul de l'accord de phase dépendant du temps. Il prend en compte la réponse atomique (dipôle, phase [6]), mais aussi la réponse macroscopique à travers la propagation du rayonnement harmonique émis et le volume dans lequel l'émission est possible.

L'approche habituelle consiste à calculer le déphasage $\varphi$ entre le rayonnement harmonique émis et le faisceau infrarouge : ce déphasage provient de l'ionisation progressive du milieu ainsi que de la phase de focalisation initiale du laser sans oublier la phase propre des harmoniques; tous ces paramètres dépendant fortement du temps [7]. Le nombre de photons émis sur l'axe par unité de temps est le résultat de l'intégrale de propagation sur la longueur de la cellule $\left(l_{\text {cell }}\right)$ [8]:

$\left|\int_{0}^{l c e l l} d_{q}(z) \exp \left(\frac{z-l_{\text {cell }}}{2 l_{a b s}}\right) \exp \left(i \varphi_{q}(z)\right) d z\right|^{2}$

où $\mathrm{d}_{\mathrm{q}}$ est le dipôle de l'harmonique $\mathrm{q}, \mathrm{l}_{\mathrm{abs}}$ est la longueur d'absorption du gaz considéré à la longueur d'onde étudiée.

Un bon accord de phase est réalisé quand le gradient de la différence de phase entre les harmoniques et le faisceau infrarouge est faible; on introduit alors la longueur de cohérence:

$l_{c o h}=\frac{\pi}{\nabla \varphi_{q}}$

Si cette longueur de cohérence s'avère constante au long de la cellule, l'intégrale précédente peut être calculée analytiquement et le problème est considérablement simplifié. Cela se produit quand 
l'intensité varie peu au sein du milieu (longueur de Rayleigh grande devant la longueur de cellule), c'est à dire pour des diaphragmes assez fermés dans notre cas. On se rapproche alors d'une configuration d'accord de phase type capillaire [2].

Notre code calcule les taux d'ionisation ADK dépendant du temps, ce qui permet d'évaluer le déphasage entre une harmonique donnée et le laser et de l'injecter dans la formule précédente. On peut alors avoir une idée de la dépendance en temps du flux harmonique. (voir figure 3):

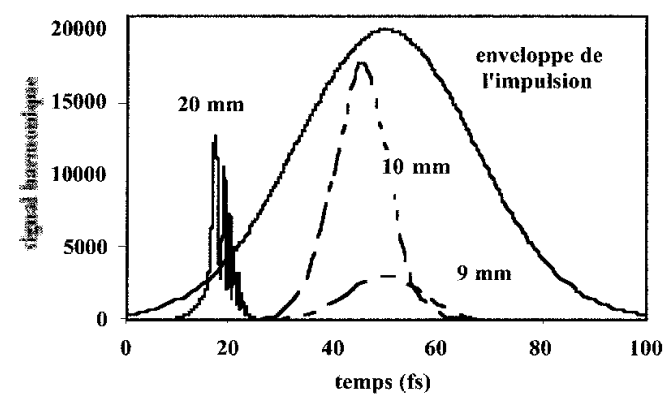

Figure 3. Flux harmonique dépendant du temps étudié pour trois tailles de diaphragme dans les conditions expérimentales de la figure 1. Le cas du diaphragme de $9 \mathrm{~mm}$ est caractéristique d'une intensité laser insuffisante : faible dipôle harmonique, émission au maximum de l'enveloppe de l'impulsion. Le cas $20 \mathrm{~mm}$ montre au contraire les effets néfastes de l'ionisation qui par dépeuplement de la population atomique et par réduction de la longueur de cohérence oblige les harmoniques à être générées en tout début d'impulsion. L'optimum résulte du compromis entre tous ces effets.

Enfin, pour reproduire la figure 1 par exemple, le signal est intégré sur toute la durée de l'impulsion et pondéré par la dimension transverse du faisceau, elle même dépendante de la taille du diaphragme.

\section{CONCLUSION}

L'utilisation d'un diaphragme sur le faisceau infrarouge pour optimiser le flux harmonique est une technique facile à mettre en œuvre et très efficace. Cependant l'effet induit sur la génération d'harmoniques est loin d'être trivial et résulte du couplage complexe entre des processus microscopiques et une réponse macroscopique du milieu.

\section{REFERENCES}

[1] S.Kazamias et al., Eur. Phys. Journal D 21, 353 (2002)

[2] C.Durfee et al, Phys. Rev, Lett. 83, (1999) 2187

[3] Y. Tamaki et al., Phys. Rev. A. 62, (2000) 063802

[4] D.Descamps et al., Phys. Rev. A 64, (2001) 031404

[5] Tyson, adaptative optics engineering handbook, (Marcel Dekker,Inc., 2000) 123

[6] M. Lewenstein et al., Phys. Rev. A 52, (1995) 4747

[7] Ph. Balcou et al. Phys. Rev. A 55, (1997) 3204

[8] E. Constant et al., Phys. Rev. Lett. 82, (1999) 1668 